\title{
Deep brain stimulation procedure complicated by intracerebral infection of DBS lead due to outbreak of COVID-19 pandemic
}

\author{
Michał Sobstyl ${ }^{1}$, Angelika Stapińska-Syniec ${ }^{1}$, Anna Kupryjaniuk ${ }^{1}$, Marcin Rylski ${ }^{2}$ \\ ${ }^{1}$ Department of Neurosurgery, Institute of Psychiatry and Neurology, Warsaw, Poland \\ ${ }^{2}$ Department of Neuroradiology, Institute of Psychiatry and Neurology, Warsaw, Poland
}

Key words: deep brain stimulation, COVID-19, intracerebral infection, coronavirus

(Neurol Neurochir Pol 2021; 55 (6): 598-600)

\section{To the Editors}

Deep brain stimulation (DBS) is a life-long treatment. Patients require scheduled follow-up visits in order to spot possible hardware-related infections [1].

The constant care of patients with DBS systems due to the COVID-19 pandemic has been almost completely stopped or been rescheduled to telemedicine, especially in the first months after the pandemic hit the world [2-5]. The fear and anxiety of infection prevented many patients with newly implanted DBS systems from reporting for skin stitches removal and subsequent scheduled follow-up visits in DBS treating experienced centres.

Herein, we report a case of early DBS hardware infection with intracerebral spread during the first month of the COVID-19 pandemic. Our aim was to present the difficulties faced by a patient with a newly implanted DBS system just before the COVID-19 pandemic with a possible life-threatening infectious complication.

A 64-year-old right-handed male with a 13-year history of PD was admitted to the neurosurgical department for a scheduled second-side staged implantation of a DBS system. The first right-sided STN (subthalamic nucleus) DBS procedure had been performed in February 2019 and resulted in a significant reduction of parkinsonian symptoms on the left side. A second stereotactic procedure was proposed, and the patient agreed and signed written informed consent before the surgery. The left-sided STN DBS procedure was scheduled for the beginning of March 2020. The STN DBS procedure was uneventful, with excellent control of parkinsonian right-sided symptoms after turning on the stimulation. In mid-March 2020, the COVID-19 pandemic began. Nearly all outpatient visits completely stopped. The skin stitches were removed later than the scheduled time limit at the patient's home. Usually, stitches after the DBS procedure are removed within 10 days after surgery at our outpatient clinic. Probably incomplete skin stitches removal resulted in superficial wound infection. The patient did not attend at the standard postoperative visit at our outpatient clinic one month after surgery. The patient did present to our clinic after two months, with signs of infection around the connector in the left parietal region. Wound debridement was carried out with relocation of the connector. A bacterial culture revealed infection caused by Proteus mirabilis. An oral antibiotic was prescribed.

Despite the antibiotic treatment, the patient was admitted to the emergency unit with visible purulent discharge from the cutaneous incision in the left parietal area (Fig. 1A). The patient was reoperated and, due to the presence of pus limited only to the connector site, it was decided not to remove the intracranial DBS electrode. The patient was treated with intravenous and subsequently with oral antibiotics for six weeks. The left-sided DBS system infection appeared to be challenged successfully.

Unfortunately, at the end of October 2020, the patient experienced chills, a headache, and a fever. The skin area around the burr hole turned red and warm. There was a slight erosion with visible purulent drainage (Fig. 1B). An emergency head CT scan revealed prominent oedema suggesting the development of a brain abscess (Fig. 1C). Urgent surgery was performed with the removal of the left DBS lead. The

Address for correspondence: Angelika Stapińska-Syniec, Department of Neurosurgery, Institute of Psychiatry and Neurology, Sobieskiego 9 Street, 02-957 Warsaw, Poland; e-mail: angelika.stapinska@gmail.com

Received: 13.06.2021 Accepted: 9.08.2021 Early publication date: 17.09.2021

This article is available in open access under Creative Common Attribution-Non-Commercial-No Derivatives 4.0 International (CC BY-NC-ND 4.0) license, allowing to download articles and share them with others as long as they credit the authors and the publisher, but without permission to change them in any way or use them commercially. 


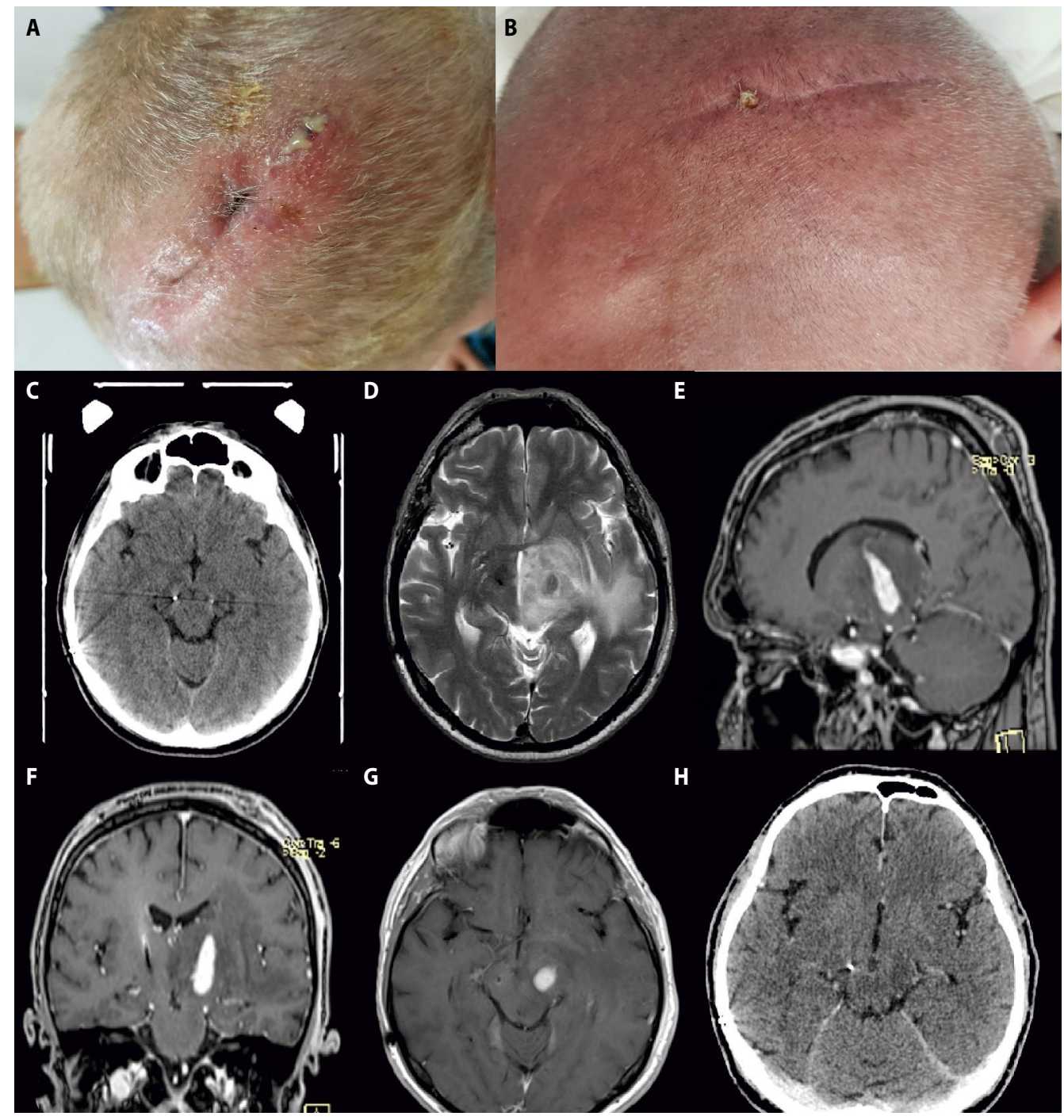

Figure 1. A. Incision site over left parietal region after failed wound debridement; B. Same site after removal of connector, small erosion at skin incision with slight purulent drainage is visible. State before left DBS electrode removal; C. Axial CT image with DBS leads placed in STN before an infection episode; D. MR axial T2-weighted image showing prominent brain parenchyma oedema around left DBS lead; E. MR parasagittal contrast-enhanced T1 weighted image indicating active brain inflammation along stereotactic trajectory; $F$. MR coronal contrast-enhanced T1 weighted image demarcating inflammation of brain parenchyma; G. MR axial contrast-enhanced T1 weighted image $4 \mathrm{~mm}$ below intercommissural plane; H. axial CT image after removal of infected DBS lead

surgery was uneventful. Postoperatively performed magnetic resonance imaging revealed prominent brain oedema visible on T2 weighted images (Fig. 1D) with active inflammation of brain parenchyma visible on contrast-enhanced $\mathrm{T} 1$ weighted images (Fig. 1 E, F, G). The patient was discharged home on the $10^{\text {th }}$ postoperative day without signs of infection and with almost normal levels of inflammatory markers. Control contrast-enhanced CT performed two months after the left DBS electrode removal revealed nearly total resolution of brain infection (Fig. 1H). Interestingly, the brain oedema resulted in good control of the right-sided PD symptoms over the follow-up period. The patient had no new neurological deficits.

This case highlights the impact of the COVID-19 pandemic on the standard care of patients with newly implanted
DBS systems. The fear of being infected, and the limited access to DBS centres, resulted in delayed skin sutures removal with subsequent early infection of DBS hardware. Infections of DBS hardware are not easy to manage and sometimes end with complete removal of the hardware [1]. In our case, revision surgeries failed and resulted in DBS hardware removal. The causative agent of the infection in the described patient was Proteus mirabilis, a less common bacterium responsible for DBS infections than the more frequently reported Staphylococcus aureus [1].

Public health guidelines have recommended that all elective medical procedures, including DBS surgery for $\mathrm{PD}$, should not be scheduled during the pandemic to prevent further virus spread and overload on healthcare systems [2]. On the other 
hand, DBS prevents the development of motor complications, and improves psychosocial functioning and the quality of life of $\mathrm{PD}$ patients [3, 4]. Another factor is that it is unknown how long the COVID-19 pandemic will last [4]. Undoubtedly, it has had, and continues to have, a profound influence on the care of patients with implanted DBS devices $[4,6]$.

COVID-19 has disrupted the standard care of patients treated with DBS worldwide. Global fear and anxiety in DBS patients prevent many of them from seeking specialised care, something which can often result in complications as described above $[4,6]$. Moreover, postponing scheduled visits to check implantable batteries may be associated with a potentially fatal risk of inducing status dystonicus or malignant withdrawal syndrome in PD [6-9].

The treatment of patients with DBS should be continuously followed to prevent complications such as infection or rapid battery depletion. DBS procedures for PD, in particular, should always be weighed against COVID-19 infection consequences and the long-term clinical benefits obtained from DBS.

Ethical permissions: Approval from the ethics review board has been waived due to the retrospective nature of the described adverse event related to the DBS procedure in our patient. The patent gave us written permission to publish his medical history as a case report.

Funding: None.

Conflicts of Interest: None.

\section{References}

1. Bhatia S, Zhang K, Oh M, et al. Infections and hardware salvage after deep brain stimulation surgery: a single-center study and review of the literature. Stereotact Funct Neurosurg. 2010; 88(3): 147-155, doi: 10.1159/000303528, indexed in Pubmed: 20357522.

2. Miocinovic S, Ostrem JL, Okun MS, et al. Recommendations for deep brain stimulation device management during a pandemic. J Parkinsons Dis. 2020; 10(3): 903-910, doi: 10.3233/JPD-202072, indexed in Pubmed: 32333552.

3. Ngoga D, Mitchell R, Kausar J, et al. Deep brain stimulation improves survival in severe Parkinson's disease. J Neurol Neurosurg Psychiatry. 2014; 85(1): 17-22, doi: 10.1136/jnnp-2012-304715, indexed in Pubmed: 23843542.

4. Bara GA, Maciaczyk J. Should deep brain stimulation programs be halted during the COVID-19 pandemic? Balancing the risk of $\mathrm{CO}$ VID-19 infection against the survival benefits of DBS. Neuromodulation. 2020; 23(8): 1222-1223, doi: 10.1111/ner.13309, indexed in Pubmed: 33181860.

5. Szmuda T, Ali S, Słoniewski P, et al. Telemedicine in neurosurgery during the novel coronavirus (COVID-19) pandemic. Neurol Neurochir Pol. 2020; 54(2): 207-208, doi: 10.5603/PJNNS.a2020.0038, indexed in Pubmed: 32319670.

6. Przytuła F, Dulski J, Sobstyl M, et al. Battery for deep brain stimulation depletion in Parkinson's Disease and dystonia patients - a systematic review. Neurol Neurochir Pol. 2021 [Epub ahead of print], doi: 10.5603/PJNNS.a2021.0041, indexed in Pubmed: 34056704.

7. Sobstyl M, Ząbek M, Kmieć T, et al. Status dystonicus due to internal pulse generator depletion in a patient with primary generalized dystonia. Mov Disord. 2014; 29(2): 188-189, doi: 10.1002/mds.25553, indexed in Pubmed: 23868498.

8. Sobstyl MR, Sławek JW, Ząbek M. The neurosurgical treatment of patients in dystonic state - overview of the literature. Neurol Neurochir Pol. 2014; 48(1): 63-70, doi: 10.1016/j.pjnns.2013.04.002, indexed in Pubmed: 24636773.

9. Rajan R, Krishnan S, Kesavapisharady KK, et al. Malignant subthalamic nucleus-deep brain stimulation withdrawal syndrome in Parkinson's disease. Mov Disord Clin Pract. 2016; 3(3): 288-291, doi: 10.1002/mdc3.12271, indexed in Pubmed: 30363553. 